\title{
FELICIDAD, PENSAMIENTO EXISTENCIALY RENDIMIENTO ACADÉMICO EN UNIVERSITARIOS DE LIMA
}

\author{
Natividad ESPÍRITU SALINAS \\ Universidad Ricardo Palma \\ natividad.espiritu@urp.edu.pe \\ José Antonio CANALES SIERRALTA \\ Universidad Ricardo Palma \\ jcanales@urp.edu.pe \\ Katherine LA TORRE CAYETANO \\ Universidad Ricardo Palma \\ klatorre@urp.edu.pe \\ William TORRES ACUÑA \\ Universidad Ricardo Palma \\ wtorres@urp.edu.pe
}

\section{RESUMEN}

Se investigó la felicidad, el pensamiento existencial y el rendimiento académico medido con el promedio histórico de notas de 264 estudiantes varones y mujeres de distintas carreras de una universidad privada de Lima. Se aplicaron la Escala de Felicidad de Alarcón (2006) y el Test de Pensamiento Existencial de Espíritu, Canales, Luna y Torres (2018). Calculando con Rho de Spearman se encontró una alta correlación positiva entre felicidad y pensamiento existencial significativa con $p<0.01$, entre la felicidad y la esencia del pensamiento existencial, así como lo espiritual, entre felicidad y rendimiento académico con $p<0.05$. Correlacionan de forma negativa significativa lo pragmático del pensamiento existencial con el rendimiento académico $p<0.01$. En el pensamiento existencial se correlacionan significativamente más alto con felicidad los ítems "Veo la vida como un regalo", "Tengo un motivo, una razón para vivir" " "Me acepto como soy".

No se encontró correlación entre la felicidad y lo pragmático en el pensamiento existencial $(r=-0.005, p=0.929)$, ni entre pensamiento existencial y rendimiento académico ( $r=$ -0.113), $p=0.067)$. Los resultados son aplicables para fines de la tutoría universitaria.

\section{PALABRAS CLAVE}

Felicidad - Pensamiento existencial - Rendimiento Académico

\section{HAPPINESS, EXISTENCIAL THOUGHT AND ACADEMIC PERFORMANCE IN UNIVERSITY STUDENTS OF LIMA}

\section{ABSTRACT}

Investigated happiness, existential thought and academic performance measured with the historical average of notes of 264 male and female students from different 
careers of a private university in Lima. Happiness Scale of Alarcón (2006) and Test of Existential Thought of Espíritu, Canales, Luna y Torres (2018) were applied. Calculating Spearman's Rho it was found a high positive correlation between happiness and significant existential thought $p<0.01$, between happiness and the essence of existential thought, as well the spiritual, between happiness and academic performance $p<0.05$. They correlate in a significant negative the pragmatic of the existential thought with the academic performance $p<0.01$. In existential thought, items "I see life as a gift", "I have a motive, a reason to live" and "I accept myself as I am" are correlated significantly higher with happiness.

No correlation was found between happiness and pragmatic in existential thought $(r$ $=-0.005, p=0.929)$, nor between existential thought and academic performance $(r=$ $-0.113), p=0.067)$. The results are applicable for university tutoring purposes.

\section{KEYWORDS}

Happiness - Existential Thought - Academic Performance

Recibido: $26 / 03 / 2020$

Aprobado: 08/06/2020

a felicidad o bienestar subjetivo (Alarcón, 2006) ha sido estudiada desde la perspectiva de la psicología positiva y es el aspecto básico del hecho de disfrutar de la vida en sí, fundamental como base sobre la cual se estructuran muchos otros procesos psicológicos, incluso supuestamente el rendimiento académico. La felicidad permite que el ser humano tenga la alegría de simplemente vivir, a pesar de todo lo negativo que pudiera experimentar en la vida. Según Alarcón la felicidad puede ser medida considerando las 4 áreas: Sentido positivo de la vida, satisfacción con la vida, realización personal y alegría de vivir.

Según Seligman, la felicidad se lograría a través de tres vías: la buena vida (pleasant life), el compromiso (engagement life) y la vida plena de sentido (meaningful life). La extroversión está asociada positivamente a la felicidad, por la mayor competencia social y la participación en actividades sociales. La felicidad se asocia con la esperanza u optimismo, el entusiasmo, la gratitud, el amar y ser amado y la curiosidad. Con respecto al pasado es importante la gratitud, el arrepentimiento y el perdón, para la resolución positiva de crisis existenciales relacionadas con experiencias traumáticas. El perdón por el mal o daño padecidos, el arrepentimiento por los ocasionados y la gratitud por los aspectos positivos de la existencia. Integrar el pasado con el presente permite una perspectiva de futuro optimista. Este optimismo es importante para el futuro, una actitud de esperanza, expectativas positivas de superación, crecimiento y maduración personal (García, 2014).

El modelo PERMA de Seligman (UIC 2013) considera P Positive Emotions (emociones positivas), E Engagement (involucramiento), R Relationship 
(relaciones), M Meaning (significado) y A Accomplishment (logro). Las emociones positivas amplían nuestros recursos intelectuales, físicos y sociales y los hacen más perdurables (Seligman, 2017).

Con respecto al pensamiento existencial, se puede decir que es la base de la vida del ser humano. Kierkegaard lo denominó "filosofía existencial" a la filosofía que no elude el más difícil de los problemas: la existencia. El "ser" ha sido tratado por obras importantes como la de Heidegger "El ser y el tiempo", Sarte "El ser y la nada", Gabriel Marcel "El misterio del ser". El existencialismo trata de mostrar la transitividad del ser, el ser como ser posible. Existir es ser un ser posible, un ser de lejanías, que se elige a sí mismo, libre, que se cuida de su ser. El hombre a diferencia de una piedra y de Dios, tiene que cuidar su ser, cuidarse de las cosas, cuidarse de sí mismo, cuidarse de los otros y ese cuidado forzoso, esa cura no le dan sosiego (Fatone, 1962). Sartre planteó que lo que el ser humano es en cada caso, su consistencia fáctica, sólo se sostiene en la asunción libre que él hace de ella, el ser humano es libre (Sartre, 2006). Llamada también Inteligencia Existencial, es un aspecto importante planteado por Howard Gardner (2005, p.4), la denominó "Inteligencia de las grandes preguntas" que se presentan cuando la persona reflexiona sobre su propia existencia y su sentido de la vida. Pero además, hay que considerar los valores que dirigirán la vida de la persona. Rokeach (1979) planteó la existencia de valores terminales referidos a estados finales de existencia y valores instrumentales o modos de conducta que servían para llegar a cumplir los valores terminales. Según Espíritu, Canales, Luna y Torres (2018) el pensamiento existencial puede ser medido explorando el motivo y la finalidad de la vida, considerando la esencia de la vida y la intencionalidad de la existencia, indagando sobre los objetivos que persigue la persona. La intencionalidad de la persona puede ser pragmática o espiritual.

Carrillo (2018) refiere que Sartre, en su obra El Existencialismo es un humanismo, planteó que el existencialista mira a la vida con optimismo, pero centrado en lo que el hombre se decida ser y hacer. El hombre es el único que no sólo es tal como él se concibe, sino tal como él se quiere, y como se concibe después de la existencia, como se quiere después de este impulso hacia la existencia; el hombre no es otra cosa que lo que él se hace. El ser humano es un proyecto que tiene que construirse permanentemente, es el ser para sí que no está acabado en cada momento de su existencia, y en eso consiste su existencia, tiene que hacerse porque es responsable de su existencia y también porque es consciente de que existe.

Para Carrillo (2018), Sartre señala que la existencia precede a la esencia y el tomar a cargo la propia existencia para nada es un planteamiento pesimista, por el contrario, es una teoría optimista debido a que no considera la existencia un determinismo sino que el ser humano puede buscar el sentido de su vida en el hacer de su existencia una respuesta al sin sentido.

El rendimiento académico universitario es importante para la consecución de los fines de formación profesional del ser y se operativiza con las notas obtenidas en la aprobación de los cursos de una carrera. Puede tener vinculación con múltiples 
variables. En la presente investigación se estudió ¿Qué relación se presenta entre la felicidad, el pensamiento existencial y el rendimiento académico en universitarios de la ciudad de Lima? y tuvo los Objetivos Generales siguientes:

OG 1 Describir la felicidad, el pensamiento existencial y el rendimiento académico en una muestra de universitarios de Lima

$\mathrm{OG}_{2}$ Relacionar la felicidad con el pensamiento existencial

$\mathrm{OG}_{3}^{2}$ Relacionar la felicidad con el rendimiento académico

$\mathrm{OG}_{4}$ Relacionar el pensamiento existencial con el rendimiento académico

Los Objetivos Específicos fueron:

$\mathrm{OE}_{1}$ Relacionar el pensamiento existencial con la felicidad: sentido positivo de la vida

$\mathrm{OE}_{2}$ Relacionar el pensamiento existencial con la felicidad: satisfacción con la vida

$\mathrm{OE}_{3}$ Relacionar el pensamiento existencial con la felicidad: realización personal

$\mathrm{OE}_{4}^{3}$ Relacionar el pensamiento existencial con la felicidad: alegría de vivir

$\mathrm{OE}_{5}$ Relacionar la felicidad: sentido positivo de la vida con el rendimiento académico

$\mathrm{OE}_{6}$ Relacionar la felicidad: satisfacción con la vida con el rendimiento académico

$\mathrm{OE}_{7}$ Relacionar la felicidad: realización personal con el rendimiento académico

$\mathrm{OE}_{8}$ Relacionar la felicidad: alegría de vivir con el rendimiento académico

Al revisar el estado del arte del problema de investigación, se encuentra que hay estudios que han tratado el tema a nivel global, del país, con la temática de la Felicidad Nacional Bruta o Gross National Happiness (GNH). Laczniak y Santos (2018) relacionaron la Felicidad Nacional Bruta con varios conceptos centrales de macromarketing como la calidad de vida, la ética, el bien común, el propósito de las actividades del mercado y los objetivos de Desarrollo Sostenible de las Naciones Unidas. La felicidad debía ser desde este punto de vista un elemento necesario para el bienestar colectivo, como una manifestación del bien común, por lo cual el propósito de la actividad empresarial del país debería percibirse con una concepción más ética y no sólo de beneficio económico.

Koky Siew (2018) plantearon que además de mejorar el nivel de la vida económica, quienes formulan las políticas públicas debían considerar las formas de aumentar la felicidad y el bienestar de la sociedad, porque éstas conducen a un mejor bienestar y mejores índices de salud de toda la población, lo que reduce la carga de asistencia sanitaria y mejora su eficacia, es decir a largo plazo reduce los costos económicos del estado.

Por otro lado, hay investigaciones en las que se demuestra que el contexto de la confianza en la política se asocia de forma positiva y significativa con la felicidad individual por ejemplo en China (Fu, 2018). Pero también hay estudios como el de Cheng, Chen, Li \& Yu (2018) que concluyen que la felicidad del pueblo chino tiene que ver más con la capacidad funcional individual de las personas que con el bienestar social o la riqueza económica de su país. Igualmente Coupe \& 
Obrizan (2018) expresaban que la situación de salud de las personas, su riqueza material y su experiencia escolar importaban mucho más que si vivían en un país de transición económica o no. De estas investigaciones se puede derivar la importancia que tiene el aspecto psicológico, incluso sobre la economía de un país. Guriev \& Melnikov (2018) indicaron que la satisfacción con la vida era impulsada principalmente por personas jóvenes, educadas y de ingresos medios, sin importar el género.

Marques, Ceinos, Silva \& Pérez (2018) encontraron que los valores de la orientación social son predictores significativos de la satisfacción con la vida. Los valores normativos más altos se asocian con una mayor satisfacción con la vida.

A nivel organizacional, las investigaciones de García, Crespo-Hervás \& Pascual (2018) concluyeron que las emociones de placer y la satisfacción eran variables de dirección que tenían una influencia importante sobre la felicidad del usuario. Giri Goswami, Pareek, Giri \& Giri (2018) plantearon que la felicidad en el lugar de trabajo crea un entorno en el que los empleados están más atentos y observan lo que sucede a su alrededor y en el entorno exterior. Factores como la transparencia en la comunicación, la construcción de relaciones positivas entre las personas, la creación de pensamientos positivos, las horas de trabajo flexibles, el equilibrio entre la vida laboral, etc. contribuyen a la construcción de una cultura organizativa positiva. Igualmente Espinoza y Paz (2018) encontraron que la felicidad organizacional tenía una correlación alta con la productividad en los trabajadores de una empresa en Arequipa. Rojas (2017) también concluyó que había correlación entre felicidad y compromiso organizacional.

A nivel educativo, Pulido-Acosta \& Herrera-Clavero (2018) encontraron que existía una relación estadísticamente significativa y directamente proporcional entre la Felicidad y el Logro Académico. Igualmente Ramírez y Fuentes (2013) concluyeron que a mayor grado de felicidad, aumentaba el grado de predicción sobre el rendimiento académico positivo de estudiantes de Ingeniería Comercial en Chile.

Por otro lado Ugarriza (2005) encontró que los alumnos de bajo rendimiento académico no esperaban conseguir lo que realmente deseaban y sentían que era muy improbable que experimentaran satisfacción en el futuro, es decir que a mayor expectativa negativa frente al futuro menor rendimiento académico.

Davey (2017) estableció una correlación entre la felicidad, la dedicación y el vigor del engagement (involucramiento) en estudiantes trabajadores de nivel superior en Lima. Ferragut y Fierro (2012) encontraron relación significativa entre bienestar e inteligencia emocional y la importancia de estos constructos psicológicos para el logro académico. Hernández (2016) concluyó que existía una correlación negativa y una probabilidad altamente significativa entre la procrastinación académica y los motivos de la procrastinación con el bienestar psicológico en estudiantes universitarios. Rosales (2017) encontró una correlación estadísticamente significativa entre la variable autoeficacia y la variable felicidad. 
Muchas causas de los problemas emocionales que afectan el rendimiento académico pueden haberse originado en la infancia de la persona. La investigación de Canales (2005) demostró que existían diferencias significativas entre los frenadores emocionales (AT Análisis Transaccional) que habían recibido en la infancia los alumnos de alto y bajo rendimiento académico. Los de bajo rendimiento académico tuvieron significativamente más frenadores emocionales implantados por las figuras parentales o sustitutos, en la infancia. El nivel intelectual no era significativamente diferente en los alumnos de alto o bajo rendimiento.

No se ha encontrado relación entre felicidad y rendimiento académico por ejemplo en la investigación de Cagle (2017), quien en su estudio de la satisfacción de vida y su efecto en el rendimiento académico de estudiantes encontró una correlación positiva demasiado pequeña para ser estadísticamente significativa. Caballero-García \& Sánchez-Ruíz (2018) mostraron resultados de que no existían diferencias significativas en la percepción de felicidad por género, edad y elección de estudios.

Port, Dianniffer Aparecida \& Thaline da (2018) al estudiar el significado de la vida encontraron que el factor más importante era la familia, además de relacionarlo con el significado de la vida y a aquellos que lo dedican. Diana Irasema, Valadez Sierra, Valdés Cuervo \& Quintana (2018) concluyeron que la autoeficacia y la orientación al logro favorecen el logro académico y que el bienestar no era una variable relacionada con este tipo de desempeño académico.

Sánchez-López, León-Hernández y Barragán-Velásquez (2015) encontraron que a mayor puntaje de inteligencia emocional se obtenía mayor puntaje en bienestar psicológico subjetivo y mayor bienestar psicológico material. A menor bienestar psicológico subjetivo hubo mayor promedio de calificaciones en el rendimiento académico, lo que sería paradógico en esa investigación.

El presente estudio plantea las siguientes hipótesis generales:

$\mathrm{HG}_{1}$ Existe relación positiva entre la felicidad y el pensamiento existencial.

$\mathrm{HG}_{2}$ Existe relación positiva entre la felicidad y el rendimiento académico.

$\mathrm{HG}_{3}$ Existe relación positiva entre el pensamiento existencial y el rendimiento académico.

Las hipótesis específicas son las siguientes:

$\mathrm{HE}_{1}$ Existe relación positiva entre el pensamiento existencial y la felicidad: Sentido positivo de la vida.

$\mathrm{HE}_{2}$ Existe relación positiva entre el pensamiento existencial y la felicidad: Satisfacción con la vida.

$\mathrm{HE}_{3}$ Existe relación positiva entre el pensamiento existencial y la felicidad: Realización personal. 
$\mathrm{HE}_{4}$ Existe relación positiva entre el pensamiento existencial y la felicidad: Alegría de vivir.

$\mathrm{HE}_{5}$ Existe relación positiva entre la felicidad: Sentido positivo de la vida y el rendimiento académico.

$\mathrm{HE}_{6}$ Existe relación positiva entre la felicidad: Satisfacción con la vida y el rendimiento académico.

$\mathrm{HE}_{7}$ Existe relación positiva entre la felicidad: Realización personal y el rendimiento académico.

$\mathrm{HE}_{8}$ Existe relación positiva entre la felicidad: Alegría de vivir y el rendimiento académico.

\section{METODOLOGÍA}

Método de investigación.- Método descriptivo.

Diseño de investigación.- Diseño descriptivo correlacional.

Población universo de estudio.- 15,000 estudiantes de una universidad privada de Lima.

Muestra.- Con un muestreo intencional, no probabilístico participaron 264 estudiantes de una universidad privada de Lima, de diversas carreras y de ambos sexos.

Instrumentos de recolección de datos.- Se utilizó un Consentimiento Informado de los alumnos para participar en la investigación. Además la Escala de Felicidad de Alarcón (2006), que mide 4 dimensiones de la Felicidad: 1 Sentido positivo de la vida, 2 Satisfacción con la vida, 3 Realización personal y 4 Alegría de vivir (Ver anexo).

El Test de Pensamiento Existencial de Espíritu, Canales, Luna y Torres (2018) que consta de 32 items (Ver anexo) y tiene un índice de confiabilidad apropiado con el Alfa de Cronbach en el área de esencia de 0.756, en el Área de Intencionalidad pragmática 0.884 y en el Área de Intencionalidad Espiritual 0.825.

Se trabajó también con el Promedio Histórico de notas de los alumnos, (para evaluar el rendimiento académico), proporcionado por la OFICIC (Oficina Central de Informática y Cómputo) de la Universidad, sobre una base de datos en Excel (elaborada por los investigadores) de los códigos de los alumnos, que habían participado en la investigación sin poner su nombre.

Técnicas de procesamiento de datos.- Se utilizó Excel y el paquete estadístico SPSS, con medidas de tendencia central, el estadístico no paramétrico de la correlación Rho de Spearman, con un nivel de significatividad de 0.05 de probabilidad. 
Procedimiento.- Los instrumentos fueron aplicados de forma grupal, en las aulas de clase, previo consentimiento informado de los estudiantes. El promedio histórico de notas fue solicitado a la autoridad académica. Los códigos que se asignaron a las carreras para el ingreso de variables al SPSS fueron los siguientes:

1. Administración y gerencia

2. Administración de negocios globales

3. Arquitectura y urbanismo

4. Biología

5. Contabilidad y finanzas

6. Derecho y ciencia política

7. Ingeniería Civil

8. Ingeniería Electrónica

9. Ingeniería Industrial

10. Ingeniería Informática

11. Ingeniería Mecatrónica

12. Marketing global y administración comercial

13. Medicina Humana

14. Medicina Veterinaria

15. Psicología

16. Traducción e interpretación

17. Turismo, hotelería y gastronomía

18. Economía

19 Ingeniería de sistemas

\section{RESULTADOS}

En la tabla 1 se presentan los datos descriptivos de los resultados obtenidos en felicidad y pensamiento existencial de los universitarios evaluados. La media obtenida en el puntaje de felicidad es 102.53. La carrera profesional que obtiene mayor puntaje en felicidad es Derecho y Ciencia Política (111.25) y la de menor puntaje en felicidad es Medicina Veterinaria (92.33). En pensamiento existencial la media de la muestra es 121.05. Marketing Global y Administración Comercial obtiene el mayor puntaje en pensamiento existencial (135.33) y Biología el menor puntaje (111.33). El promedio histórico de notas en toda la muestra fue de 12.78 en un sistema vigesimal de evaluación, siendo el puntaje mínimo 07 y el máximo 18. 
Tabla 1. Media y Desviación Estándar del Pensamiento Existencial y de Felicidad en estudiantes de diferentes carreras profesionales de una universidad privada de Lima

\begin{tabular}{|c|c|c|c|c|c|c|c|c|c|c|}
\hline \multirow[b]{2}{*}{$\begin{array}{l}\text { Carrera } \\
\text { Profesional } \\
\text { del alumno }\end{array}$} & \multirow[b]{2}{*}{$\begin{array}{l}\text { Estadís- } \\
\text { ticos }\end{array}$} & \multicolumn{4}{|c|}{ Pensamiento Existencial } & \multicolumn{5}{|c|}{ Felicidad } \\
\hline & & $\begin{array}{l}\text { Esen- } \\
\text { cia }\end{array}$ & $\begin{array}{r}\text { Pragmá- } \\
\text { tico }\end{array}$ & $\begin{array}{r}\text { Espiri- } \\
\text { tual }\end{array}$ & $\begin{array}{c}\text { Puntaje } \\
\text { Total }\end{array}$ & $\begin{array}{r}\text { Senti- } \\
\text { do Po- } \\
\text { sitivo } \\
\text { de la } \\
\text { Vida } \\
\text { F1 }\end{array}$ & $\begin{array}{r}\text { Satisfac- } \\
\text { ción con } \\
\text { la Vida } \\
\text { F2 }\end{array}$ & $\begin{array}{r}\text { Realiza- } \\
\text { ción } \\
\text { Personal } \\
\text { F3 }\end{array}$ & $\begin{array}{r}\text { Alegría } \\
\text { de } \\
\text { Vivir } \\
\text { F4 }\end{array}$ & $\begin{array}{c}\text { Puntaje } \\
\text { Total }\end{array}$ \\
\hline \multirow{3}{*}{$\begin{array}{l}\text { Adminis- } \\
\text { tración } \\
\text { y Gerencia }\end{array}$} & Media & 36.53 & 37.47 & 50.00 & 124.00 & 44.47 & 23.12 & 20.47 & 16.18 & 104.24 \\
\hline & $\mathrm{N}$ & 17 & 17 & 17 & 17 & 17 & 17 & 17 & 17 & 17 \\
\hline & $\begin{array}{l}\text { Desviación } \\
\text { Estándar }\end{array}$ & 4.317 & 5.864 & 5.809 & 14.195 & 5.959 & 3.257 & 3.923 & 2.215 & 12.657 \\
\hline \multirow{3}{*}{$\begin{array}{l}\text { Adminis- } \\
\text { tración de } \\
\text { Negocios } \\
\text { Globales }\end{array}$} & Media & 35.64 & 34.55 & 51.36 & 121.55 & 41.00 & 22.55 & 21.27 & 16.45 & 101.27 \\
\hline & $\mathrm{N}$ & 11 & 11 & 11 & 11 & 11 & 11 & 11 & 11 & 11 \\
\hline & $\begin{array}{l}\text { Desviación } \\
\text { Estándar }\end{array}$ & 4.032 & 6.023 & 3.776 & 8.190 & 9.338 & 3.174 & 3.952 & 1.968 & 15.173 \\
\hline \multirow{3}{*}{$\begin{array}{l}\text { Arquitec- } \\
\text { tura y } \\
\text { Urbanismo }\end{array}$} & Media & 35.39 & 36.22 & 51.68 & 123.29 & 44.93 & 22.88 & 20.37 & 16.49 & 104.66 \\
\hline & $\mathrm{N}$ & 41 & 41 & 41 & 41 & 41 & 41 & 41 & 41 & 41 \\
\hline & $\begin{array}{l}\text { Desviación } \\
\text { Estándar }\end{array}$ & 3.980 & 6.471 & 6.223 & 13.735 & 6.498 & 2.786 & 3.813 & 1.899 & 12.241 \\
\hline \multirow[t]{3}{*}{ Biología } & Media & 31.67 & 31.67 & 48.00 & 111.33 & 45.00 & 19.67 & 18.67 & 16.67 & 100.00 \\
\hline & $\mathrm{N}$ & 3 & 3 & 3 & 3 & 3 & 3 & 3 & 3 & 3 \\
\hline & $\begin{array}{l}\text { Desviación } \\
\text { Estándar }\end{array}$ & 8.145 & 4.041 & 8.185 & 19.630 & 3.000 & 4.726 & 5.508 & 3.512 & 15.875 \\
\hline \multirow{3}{*}{$\begin{array}{l}\text { Contabili- } \\
\text { dady } \\
\text { Finanzas }\end{array}$} & Media & 35.71 & 37.00 & 51.29 & 124.00 & 42.86 & 21.57 & 19.29 & 16.14 & 99.86 \\
\hline & $\mathrm{N}$ & 7 & 7 & 7 & 7 & 7 & 7 & 7 & 7 & 7 \\
\hline & $\begin{array}{l}\text { Desviación } \\
\text { Estándar }\end{array}$ & 4.112 & 4.435 & 2.430 & 8.718 & 7.841 & 4.036 & 3.729 & 1.574 & 13.171 \\
\hline \multirow{3}{*}{$\begin{array}{l}\text { Derecho y } \\
\text { Ciencia } \\
\text { Política }\end{array}$} & Media & 38.25 & 35.75 & 56.00 & 130.00 & 47.00 & 24.50 & 22.25 & 17.50 & 111.25 \\
\hline & N & 4 & 4 & 4 & 4 & 4 & 4 & 4 & 4 & 4 \\
\hline & $\begin{array}{l}\text { Desviación } \\
\text { Estándar }\end{array}$ & 1.708 & 8.958 & 4.163 & 13.614 & 4.967 & 3.109 & 3.403 & 1.732 & 12.121 \\
\hline \multirow{3}{*}{$\begin{array}{l}\text { Ingeniería } \\
\text { Civil }\end{array}$} & Media & 34.38 & 35.54 & 50.46 & 120.38 & 42.13 & 22.17 & 18.96 & 16.63 & 99.87 \\
\hline & $\mathrm{N}$ & 23 & 23 & 23 & 23 & 23 & 23 & 23 & 23 & 23 \\
\hline & $\begin{array}{l}\text { Desviación } \\
\text { Estándar }\end{array}$ & 3.728 & 4.818 & 5.316 & 11.228 & 5.736 & 3.571 & 3.816 & 1.907 & 11.863 \\
\hline \multirow{3}{*}{$\begin{array}{l}\text { Ingeniería } \\
\text { Electrónica }\end{array}$} & Media & 39.33 & 36.00 & 53.67 & 129.00 & 45.67 & 23.33 & 20.67 & 16.00 & 105.67 \\
\hline & $\mathrm{N}$ & 3 & 3 & 3 & 3 & 3 & 3 & 3 & 3 & 3 \\
\hline & $\begin{array}{l}\text { Desviación } \\
\text { Estándar }\end{array}$ & 3.055 & 4.359 & 5.508 & 10.000 & 4.041 & 3.055 & 5.508 & 2.000 & 13.614 \\
\hline \multirow{3}{*}{$\begin{array}{l}\text { Ingeniería } \\
\text { Industrial }\end{array}$} & Media & 37.73 & 34.55 & 52.82 & 125.09 & 45.73 & 24.64 & 22.45 & 17.18 & 110.00 \\
\hline & $\mathrm{N}$ & 11 & 11 & 11 & 11 & 11 & 11 & 11 & 11 & 11 \\
\hline & $\begin{array}{l}\text { Desviación } \\
\text { Estándar }\end{array}$ & 4.338 & 6.393 & 5.528 & 13.824 & 5.934 & 3.414 & 1.635 & 2.523 & 11.419 \\
\hline
\end{tabular}




\begin{tabular}{|c|c|c|c|c|c|c|c|c|c|c|}
\hline \multirow{3}{*}{$\begin{array}{l}\text { Ingeniería } \\
\text { Informática }\end{array}$} & Media & 31.63 & 33.25 & 45.62 & 110.50 & 37.75 & 19.63 & 19.75 & 16.25 & 93.38 \\
\hline & $\mathrm{N}$ & 8 & 8 & 8 & 8 & 8 & 8 & 8 & 8 & 8 \\
\hline & $\begin{array}{l}\text { Desviación } \\
\text { Estándar }\end{array}$ & 6.346 & 7.459 & 10.336 & 21.889 & 5.523 & 1.506 & 2.188 & 2.053 & 8.141 \\
\hline \multirow{3}{*}{$\begin{array}{l}\text { Ingeniería } \\
\text { Mecatró- } \\
\text { nica }\end{array}$} & Media & 37.33 & 38.67 & 55.00 & 131.00 & 46.00 & 23.00 & 20.33 & 16.67 & 106.00 \\
\hline & $\mathrm{N}$ & 3 & 3 & 3 & 3 & 3 & 3 & 3 & 3 & 3 \\
\hline & $\begin{array}{l}\text { Desviación } \\
\text { Estándar }\end{array}$ & 1.155 & 2.082 & 2.646 & 5.292 & 9.539 & 4.583 & 4.509 & 1.528 & 19.313 \\
\hline \multirow{3}{*}{$\begin{array}{l}\text { Marketing } \\
\text { Global y } \\
\text { Adminis- } \\
\text { tración } \\
\text { Comercial }\end{array}$} & Media & 36.67 & 42.67 & 56.00 & 135.33 & 41.00 & 21.00 & 19.67 & 14.33 & 96.00 \\
\hline & $\mathrm{N}$ & 3 & 3 & 3 & 3 & 3 & 3 & 3 & 3 & 3 \\
\hline & $\begin{array}{l}\text { Desviación } \\
\text { Estándar }\end{array}$ & 2.517 & 7.024 & 6.000 & 14.572 & 8.185 & 3.606 & 2.517 & 2.082 & 15.588 \\
\hline \multirow{4}{*}{$\begin{array}{l}\text { Medicina } \\
\text { Humana }\end{array}$} & Media & 35.50 & 32.50 & 49.13 & 117.13 & 44.13 & 23.50 & 21.12 & 17.25 & 106.00 \\
\hline & $\mathrm{N}$ & 16 & 16 & 16 & 16 & 16 & 16 & 16 & 16 & 16 \\
\hline & Desviación & & & & & & & & & \\
\hline & Estándar & 4.000 & 5.190 & 4.801 & 10.039 & 5.476 & 4.351 & 4.349 & 1.983 & 14.579 \\
\hline \multirow{4}{*}{$\begin{array}{l}\text { Medicina } \\
\text { Veterinaria }\end{array}$} & Media & 33.40 & 40.53 & 52.40 & 126.33 & 37.20 & 20.67 & 19.47 & 15.00 & 92.33 \\
\hline & $\mathrm{N}$ & 14 & 14 & 14 & 14 & 14 & 14 & 14 & 14 & 14 \\
\hline & Desviación & & & & & & & & & \\
\hline & Estándar & 5.180 & 7.963 & 5.329 & 13.788 & 11.620 & 4.761 & 3.603 & 2.478 & 20.166 \\
\hline \multirow[t]{3}{*}{ Psicología } & Media & 35.35 & 32.94 & 49.45 & 117.73 & 44.58 & 22.14 & 20.65 & 16.23 & 103.60 \\
\hline & $\mathrm{N}$ & 78 & 78 & 78 & 78 & 78 & 78 & 78 & 78 & 78 \\
\hline & $\begin{array}{l}\text { Desviación } \\
\text { Estándar }\end{array}$ & 4.941 & 5.990 & 5.826 & 12.340 & 7.138 & 3.440 & 3.692 & 2.568 & 14.539 \\
\hline \multirow{3}{*}{$\begin{array}{l}\text { Traducción e } \\
\text { Interpretación }\end{array}$} & Media & 33.33 & 35.25 & 48.33 & 116.92 & 42.00 & 21.50 & 19.17 & 15.00 & 97.67 \\
\hline & $\mathrm{N}$ & 12 & 12 & 12 & 12 & 12 & 12 & 12 & 12 & 12 \\
\hline & $\begin{array}{l}\text { Desviación } \\
\text { Estándar }\end{array}$ & 6.485 & 5.578 & 4.735 & 11.131 & 9.361 & 6.127 & 4.086 & 2.763 & 20.716 \\
\hline \multirow{3}{*}{$\begin{array}{l}\text { Turismo, } \\
\text { Hotelería y } \\
\text { Gastronomía }\end{array}$} & Media & 31.00 & 39.00 & 45.00 & 115.00 & 43.00 & 22.00 & 22.00 & 16.00 & 103.00 \\
\hline & $\mathrm{N}$ & 1 & 1 & 1 & 1 & 1 & 1 & 1 & 1 & 1 \\
\hline & Media & 36.25 & 38.13 & 57.25 & 131.63 & 44.37 & 23.25 & 20.25 & 15.88 & 103.75 \\
\hline \multirow[t]{2}{*}{ Economía } & $N$ & 8 & 8 & 8 & 8 & 8 & 8 & 8 & 8 & 8 \\
\hline & $\begin{array}{l}\text { Desviación } \\
\text { Estándar }\end{array}$ & 2.121 & 5.987 & 16.456 & 20.976 & 7.891 & 1.669 & 4.062 & 1.356 & 10.306 \\
\hline \multirow{3}{*}{$\begin{array}{l}\text { Ingeniería de } \\
\text { Sistemas }\end{array}$} & Media & 36.00 & 31.00 & 53.00 & 120.00 & 49.00 & 23.00 & 19.00 & 18.00 & 109.00 \\
\hline & $\mathrm{N}$ & 1 & 1 & 1 & 1 & 1 & 1 & 1 & 1 & 1 \\
\hline & Media & 35.26 & 35.13 & 50.67 & 121.05 & 43.53 & 22.37 & 20.35 & 16.29 & 102.53 \\
\hline \multirow[t]{2}{*}{ Total } & $N$ & 264 & 264 & 264 & 264 & 264 & 264 & 264 & 264 & 264 \\
\hline & $\begin{array}{l}\text { Desviación } \\
\text { Estándar }\end{array}$ & 4.614 & 6.320 & 6.462 & 13.497 & 7.352 & 3.627 & 3.725 & 2.261 & 14.344 \\
\hline
\end{tabular}

Con respecto a la Hipótesis General 1: Existe relación positiva entre la felicidad y el pensamiento existencial, se ha encontrado un coeficiente de correlación positivo Rho de Spearman de 0.374 , que es significativo al 0.01 de probabilidad, por lo que se comprueba esta hipótesis (tabla 2). Cuando se relaciona la felicidad con el pensamiento existencial, en el área de Esencia (tabla 2), se encuentra una alta 
correlación positiva de 0.688 , significativa al 0.01 . Igualmente se observa una alta correlación positiva entre felicidad y lo espiritual del pensamiento existencial. Al relacionar lo Pragmático del Pensamiento existencial con la felicidad, no se ha encontrado una correlación significativa, el puntaje de la Rho de Spearman es de -0.005 con una $p$ de 0.929 expresado en ítems tales como la vida tiene como principal objetivo tener casas, tener autos, hacer viajes, etc.

Tabla 2. Cálculo de la relación entre la Felicidad y el Pensamiento Existencial en estudiantes de una universidad privada de Lima, utilizando el coeficiente de correlación de Spearman

\begin{tabular}{lccc}
\hline & Felicidad & $\mathrm{N}$ & $\mathrm{p}$ \\
\hline Pensamiento existencial & $0.374^{* *}$ & 264 & 0.000 \\
Esencia del Pensamiento Existencial & $0.688^{* *}$ & 264 & 0.000 \\
Espiritual del Pensamiento Existencial & $0.324^{* *}$ & 264 & 0.000 \\
Pragmático del Pensamiento Existencial & -0.005 & 264 & 0.929 \\
\hline$* * \mathrm{p}<0.01$ & & &
\end{tabular}

Al haberse establecido que el área de Esencia del pensamiento existencial es la que tiene una mayor correlación, complementariamente a los objetivos de la investigación se analizaron los 9 items que contiene esta área, encontrándose que "Veo la vida como un regalo" (ítem 3), "Tengo un motivo, una razón para vivir" (ítem 4) y "Me acepto como soy" (ítem 8) tienen los puntajes más altos en correlación, según se aprecia en la tabla 3, aunque todos los ítems son significativamente relacionados positivamente con felicidad, con una probabilidad al 0.01 .

Tabla 3. Cálculo de la relación entre la Felicidad y los ítems del área Esencia del Pensamiento Existencial en estudiantes de una universidad privada de Lima, utilizando el coeficiente de correlación de Spearman

\begin{tabular}{llll}
\hline $\begin{array}{l}\text { Test de Pensamiento Existencial de Espíritu, } \\
\text { Canales, Luna }\end{array}$ & $\begin{array}{c}\text { Felicidad, } \\
\text { Reynaldo } \\
\text { y Torres, área Esencia }\end{array}$ & N & p \\
\hline Item 1 "El pasado me preocupa" (-) & $0.341^{* *}$ & 264 & 0.00 \\
Item 2 "Me concentro en el presente" & $0.323^{* *}$ & 264 & 0.00 \\
Item 3 "Veo la vida como un regalo" & $0.503^{* *}$ & 264 & 0.00 \\
Item 4 "Tengo un motivo, una razón para vivir" & $0.526^{* *}$ & 264 & 0.00 \\
Item 5 "Me concentro en el aspecto bello de la & $0.487^{* *}$ & 264 & 0.00 \\
$\quad$ vida cotidiana" & & & \\
Item 6 "Disfruto de la meditación" & $0.197^{* *}$ & 264 & 0.001 \\
Item 7 "Mis actividades las relaciono con un & $0.309^{* *}$ & 264 & 0.00 \\
$\quad$ propósito trascendental" & & \\
Item 8 "Me acepto como soy" & $0.547^{* *}$ & 264 & 0.00 \\
Item 9 "Trato de aprovechar cada momento" & $0.337^{* *}$ & 264 & 0.00 \\
\hline **p $<0.01$ & & &
\end{tabular}


La hipótesis $\mathrm{HG}_{2:}$ Existe relación positiva entre la felicidad y el rendimiento académico se comprueba una correlación positiva significativa con $p<0.05$, según la tabla 4. La $\mathrm{HE}_{5}$ Existe relación positiva entre la felicidad: Sentido positivo de la vida y el rendimiento académico, se comprueba una correlación positiva significativa con $\mathrm{p}<0.05$. La $\mathrm{HE}_{6}$ Existe relación positiva entre la felicidad: Satisfacción con la vida y el rendimiento académico, no es comprobada con $r=$ 0.088 que no es significativa con $p=0.153$. La $\mathrm{HE}_{7}$ Existe relación positiva entre la felicidad: Realización personal y el rendimiento académico, se comprueba con una correlación positiva significativa con $\mathrm{p}<0.05$. $\mathrm{La} \mathrm{HE}_{8}$ Existe relación positiva entre la felicidad: Alegría de vivir y el rendimiento académico, no se comprueba con $r=-0.030$ y una $p=0.628$ que no resulta significativa. La hipótesis $\mathrm{HG}_{3}$ : Existe relación positiva entre el pensamiento existencial y el rendimiento académico, no es comprobada, con un puntaje $r=0.113$ y una $p$ de 0.067 . Sin embargo, al realizar el cálculo de la correlación de Spearman entre lo Pragmático del Pensamiento Existencial con el Rendimiento Académico, se encontró una $p<0.01$ con una alta correlación negativa, es decir a mayor Pragmatismo, menor Rendimiento Académico.

Tabla 4. Cálculo de la relación entre Felicidad y Rendimiento Académico en estudiantes de una universidad privada de Lima, utilizando el coeficiente de correlación de Spearman

\begin{tabular}{lccc}
\hline & Rendimiento Académico & $\mathrm{N}$ & $\mathrm{p}$ \\
\hline Felicidad & $0.126^{*}$ & 264 & 0.041 \\
Felicidad: sentido positivo de la vida & $0.125^{*}$ & 264 & 0.043 \\
Felicidad: satisfacción con la vida & 0.088 & 264 & 0.153 \\
Felicidad: realización personal & $0.132^{*}$ & 264 & 0.032 \\
Felicidad: alegría de vivir & -0.030 & 264 & 0.628 \\
Pensamiento Existencial & -0.113 & 264 & 0.067 \\
Pragmático del Pensamiento & $-0.218^{* *}$ & 264 & 0.000 \\
Existencial & & & \\
\hline
\end{tabular}

${ }^{*} \mathrm{p}<0.05$

${ }^{* *} \mathrm{p}<0.01$

Con respecto a la hipótesis específica, $\mathrm{HE}$, : Existe relación positiva entre el pensamiento existencial y la felicidad: Sentido positivo de la vida, se comprueba una correlación positiva significativa con una $\mathrm{p}<0.01$ según la tabla 5 . La $\mathrm{HE}_{2}$ Existe relación positiva entre el pensamiento existencial y la felicidad: Satisfacción con la vida, es comprobada con una correlación positiva significativa con $\mathrm{p}<$ 0.01. Igualmente la $\mathrm{HE}_{3}$ Existe relación positiva entre el pensamiento existencial y la felicidad: Realización personal, es comprobada con una correlación positiva significativa con $\mathrm{p}<0.01$. La HE ${ }_{4}$ Existe relación positiva entre el pensamiento existencial y la felicidad: Alegría de vivir, se comprueba una correlación positiva significativa con $p<0.01$. 
Tabla 5. Cálculo de la relación entre el Pensamiento Existencial y la Felicidad: sentido positivo de la vida en estudiantes de una universidad privada de Lima, utilizando el coeficiente de correlación de Spearman

\begin{tabular}{lccc}
\hline & Pensamiento Existencial & $\mathrm{N}$ & $\mathrm{p}$ \\
\hline Felicidad: sentido positivo de la vida & $0.254^{* *}$ & 264 & 0.000 \\
Felicidad: satisfacción con la vida & $0.418^{* *}$ & 264 & 0.000 \\
Felicidad: realización personal & $0.356^{* *}$ & 264 & 0.000 \\
Felicidad: alegría de vivir & $0.345^{* *}$ & 264 & 0.000 \\
\hline
\end{tabular}

*** $p<0.01$

\section{DISCUSIÓN}

La Felicidad como bienestar subjetivo y el Pensamiento Existencial se encuentran muy relacionados en los resultados de esta investigación (Hipótesis General 1, tabla 2). Podríamos afirmar que los universitarios más felices tienen resuelto su problema existencial del ser. Esto corrobora la teoría de Seligman, según la cual la felicidad se lograría a través de tres vías: la buena vida (pleasant life), el compromiso (engagement life) y especialmente en lo referente a la vida plena de sentido (meaningful life), que está relacionada con el pensamiento existencial (García, 2014).

Los items del Test de Pensamiento Existencial de Espíritu, Canales, Luna y Torres (2018) en el área de Esencia se correlacionaron positivamente al 0.01 de significatividad (tabla 3) con el Test de Felicidad de Alarcón (2006). Se encontró alta relación positiva entre el pensamiento existencial y el "sentido positivo de la vida" de la felicidad con $\mathrm{p}<0.01$ (hipótesis específica 1, tabla 5). Existe relación positiva entre el pensamiento existencial y la "satisfacción con la vida" de la felicidad, con $p<0.01$ (hipótesis específica 2, tabla 5). Se encontró también relación positiva entre el pensamiento existencial y la "realización personal" de la felicidad, con $p<0.01$ (hipótesis específica 3, tabla 5). Y existe relación positiva entre el pensamiento existencial y la "alegría de vivir" de la felicidad, con $p<0.01$ (hipótesis específica 4, tabla 5). Se debe aconsejar a los alumnos universitarios que consideren los siguientes pensamientos existenciales positivos que se relacionan más $(p<0.01)$ con la felicidad:

1. Veo la vida como un regalo

2. Tengo un motivo, una razón para vivir

3. Me acepto como soy

Pero además, también con $\mathrm{p}<0.01$, partiendo de los resultados de los items del Test de Pensamiento Existencial de Espíritu, Canales, Luna y Torres (2018) se puede recomendar a nivel tutorial universitario estos aspectos que se relacionan positivamente con la felicidad: No preocuparse por el pasado (ítem 1), concentrarse en el presente (ítem 2), aprovechar cada momento (ítem 9), concentrarse en el aspecto bello de la vida cotidiana (ítem 5), disfrutar de la meditación (ítem 6) y relacionar las actividades con un propósito trascendental (ítem 7). 
Una persona que está en crisis existencial no puede ser feliz. Se necesita la tranquilidad de tener resuelta la existencia para estar feliz. En la muestra de la investigación el Pragmatismo no se relaciona con la felicidad, lo que podría decirnos que el deseo de tener casas, autos, etc. no está vinculado con la percepción de la felicidad, que es un constructo muy subjetivo. La gente más feliz desea tener en la vida aspectos más espirituales y no sólo lo material, lo que concuerda con los resultados de la investigación de Cheng, Chen, Li \& Yu (2018), quienes concluyeron que la felicidad del pueblo chino tiene que ver más con factores individuales que con la riqueza económica de su país. Así mismo Marquez, Ceinos, Silva \& Perez (2018) habían encontrado que los valores de la orientación social son predictores significativos de la satisfacción con la vida. Los valores normativos más altos se asocian con una mayor satisfacción con la vida.

La felicidad se relaciona positivamente con $\mathrm{p}<0.05$ con el rendimiento académico (hipótesis general 2) expresado en el promedio histórico de notas de los alumnos universitarios. El mayor rendimiento académico está asociado a la mayor felicidad. ¿El buen rendimiento académico es causa de la felicidad experimentada o la felicidad causa un buen rendimiento académico?. En la presente investigación no se determina que una variable sea la causa de la otra, sólo se encuentra una correlación positiva significativa.

Esta investigación concuerda con los resultados de Coupe (2018), Cheng (2018), Davey (2017), Ferragut y Fierro (2012), Giri (2018), Hernandez (2016), Pulido (2018), Ramírez y Fuentes (2018) y Rosales (2017) que encontraron relación positiva y significativa entre la felicidad y el rendimiento académico. A nivel organizacional Espinoza y Paz (2018) y Rojas (2017) habían encontrado también relación positiva entre la felicidad y el rendimiento organizacional del personal.

Ugarriza (2005), en su investigación concluyó que cuando los alumnos tenían expectativas negativas frente a su futuro, tenían también menor rendimiento académico. En la investigación de Canales (2005) los universitarios que tenían menor rendimiento académico tenían más problemas emocionales implantados por las figuras parentales como frenadores en su infancia. Con la presente investigación se podría agregar que también son menos felices.

Los hallazgos de esta investigación contradicen los encontrados por aquellas en las que no existía relación entre la felicidad y el rendimiento académico, como es el caso de Caballero y García (2018), Cagle (2017), Port (2018) y Diana Irasema (2018).

Pero haciendo un balance general de los estudios sobre la felicidad en las diferentes investigaciones a nivel global, se puede afirmar que la felicidad sí se relaciona positivamente con el rendimiento académico, lo cual es muy importante para fines de la tutoría universitaria, con el objetivo de incrementar el rendimiento académico en los alumnos de educación superior. Estos datos deberían ser tomados en cuenta en Perú por la SUNEDU (Superintendencia Nacional de Educación Superior Universitaria). 
También sería importante explorar, para futuras investigaciones, las causas de los bajos niveles de felicidad en los universitarios. ¿Es el fracaso en el rendimiento académico lo que genera un menor nivel de felicidad o son motivos de tipo familiar, de pareja, problemas económicos, de hábitos de estudio, de no tener metas académicas, etc.? Para ello habría que correlacionar esas variables con la felicidad de los alumnos. Se podrían evaluar los "problemas personales" de los universitarios correlacionándolos con la Escala de Felicidad de Alarcón (2006), así como con el Test de Pensamiento Existencial de Espíritu, Canales, Luna y Torres (2018).

Con respecto a las hipótesis específicas que relacionan el rendimiento académico con la felicidad se encuentra correlaciones positivas significativas: $p<0.05$ entre rendimiento académico y "sentido positivo" de la felicidad (hipótesis específica 5 , tabla 4), p< 0.05 entre rendimiento académico y "realización personal" de la felicidad (hipótesis específica 7, tabla 4).

No se comprueba la hipótesis específica 6 sobre relación entre la felicidad: satisfacción con la vida y el rendimiento académico ( $p=0.153$, tabla 4). Igualmente no se comprueba la hipótesis específica 8 , es decir no hay relación entre rendimiento académico y "alegría de vivir" de la felicidad, pues la $p=0.628$ no es significativa (tabla 4).

Por otro lado, se debe trabajar a nivel tutorial universitario el tema del Pragmatismo en el pensamiento existencial en los estudiantes de menor rendimiento académico. En la investigación se encontró un alto Pragmatismo: tener casas, autos, etc. correlacionado negativamente con el bajo rendimiento académico $p<0.01$ (tabla 4). Se debe aconsejar a los alumnos que si quieren casas, autos... placeres de la vida, una buena forma de conseguirlos es mediante su futuro trabajo con una carrera profesional estudiada en la universidad, porque la persona estará más capacitada y puede ser más competitiva en un mundo que exige la tecnificación, la especialización. Un título profesional facilita la inserción laboral en la sociedad. Pero las metas a largo plazo en las personas "Pragmáticas" están muy distantes, por eso es necesario hacerles comprender que deben trazarse objetivos más cortos y cotidianos, relacionados con los hábitos de estudio en la vida diaria, los valores instrumentales que llevan a los valores terminales (Rockeach, 1979), para que puedan alcanzar primero la meta de aprobar los cursos en un semestre académico y así darle un sentido de un plan de vida orientado al futuro, no una existencia que esté a la deriva, al azar, o sólo situada en el "ahora" y la satisfacción de los placeres de la vida: el hedonismo.

El pensamiento existencial, tanto en Esencia como en lo Espiritual, en esta investigación no está relacionado significativamente con el rendimiento académico (hipótesis general 3 , tabla $4, r=-0.113$ con $p=0.067$ ). Es decir, los dilemas existenciales pueden estar presentes tanto en alumnos de bajo, como de alto rendimiento académico. El rendimiento académico está más correlacionado positivamente con la felicidad que con el pensamiento existencial de los alumnos.

En la felicidad Seligman había planteado el modelo PERMA, Positive Emotions, Engagement, Relationship, Meaning and Accomplishment (UIC, 2013), lo que 
explicaría los resultados obtenidos. No hay duda de que las emociones positivas amplían los recursos intelectuales de la persona (Seligman, 2017).

Si bien es cierto, no fue objetivo de esta investigación comparar los niveles de felicidad de los estudiantes por carreras, sin embargo es interesante notar que los alumnos de la carrera de Derecho y Ciencia Política obtuvieron el mayor puntaje de felicidad (111.28) y los de Medicina Veterinaria el menor puntaje (92.33). Con respecto al Pensamiento Existencial, Marketing global y Administración Comercial fue la carrera de estudios que obtuvo el mayor puntaje (135.33) y Biología el menor puntaje (111.33). Estos datos podrían servir de base para el planteamiento de futuros problemas de investigación.

\section{REFERENCIAS}

Alarcón, R. (2006). Desarrollo de una Escala Factorial para Medir la Felicidad. Revista Interamericana de Psicología. 40(1).

Caballero-García, P.A. \& Sánchez-Ruíz, S. (2018). La felicidad en estudiantes universitarios. ¿Existen diferencias según género, edad o elección de estudios? Revista Electrónica Interuniversitaria de Formación del Profesorado, 21(3), 1-18.

Canales, J. A. (2005). Estudio Comparativo de las Conductas Parentales Negativas entre Estudiantes Universitarios de Psicología de Alto y Bajo Rendimiento Académico a través del Análisis Transaccional. (Tesis de Maestro en Docencia Superior), Universidad Ricardo Palma, Perú.

Carrillo, R. (2018). El sentido filosófico de la vida en el pensamiento existencialista: Una lectura desde Ellacuría. Teoría y Praxis, (32), 29-44. Consultado en: https:// doi.org/10.5377/typ.v0i32.6390

Coupe, T., \& Obrizan, M. (2018). Adolescents' (un)happiness in transition. Journal of Comparative Economics, 46(3), 858-873. https://doi.org/10.1016/j. jce.2018.07.012

Cheng, H., Chen, C., Li, D., \& Yu, H. (2018). The Mystery of Chinese People's Happiness. Journal of Happiness Studies, 19(7), 2095-2114. https://doi. org/10.1007/s10902-017-9912-8

Cagle, J. (2017). A study of the life satisfaction of students and its effect on their academic achievement. (Tesis para Doctorado) Carson- Newman University.

Davey, K. (2017). Felicidad y Engagement en estudiantes-trabajadores de nivel superior en Lima. Revista de Investigación en Psicología, 20, 2, pp. 389-406.

Diana Irasema, C. A., Valadez Sierra, M. D., Valdés Cuervo, A. A., \& Quintana, J. T. (2018). Diferencias en autoeficacia académica, bienestar psicológico y motivación al logro en estudiantes universitarios con alto y bajo desempeño académico. Psicología Desde El Caribe, 35(1) Consultado en https://search. proquest.com/docview/2021779987?accountid=45097 
Espinoza, M. y Paz, K. (2018). Influencia de la felicidad organizacional en la productividad de los colaboradores en los centros de atención al cliente de la empresa América Móvil - Claro, Arequipa, 2016 - 2017. Tesis de pre grado en Administración de Negocios. Universidad Católica San Pablo, Perú.

Espíritu, N., Canales, J., Luna, M. y Torres, W. (2018). Elaboración del Test de Pensamiento Crítico Existencial de Lima. Centro de Investigación de la Universidad Ricardo Palma CIURP. Lima (en prensa).

Fatone, V. (1962). Introducción al existencialismo. Argentina: Columba. Consultado en https://es.scribd.com/doc/221623868/Fatone-Vicente-Introduccion-AlExistencialismo-pdf

Ferragut, M. y Fierro, A. (2012). Inteligencia emocional, bienestar personal y rendimiento académico en preadolescentes. Revista Latinoamericana de Psicología. 44, 3, pp. 95-104.

Fu, X. (2018). The Contextual Effects of Political Trust on Happiness: Evidence from China. Social Indicators Research, 139(2), 491-516. https://doi.org/10.1007/ s11205-017-1721-2

García, N. M., Crespo-Hervás, J., \& Pascual, F. G. (2018). Predictive variables of happiness in private sports centres. Journal of Physical Education and Sport, 18, 1391-1401. doi: http://dx.doi.org/10.7752/jpes.2018.s3206

García, J. (2014). Psicología positiva, bienestar y calidad de vida. En-claves del Pensamiento, vol. VIII, núm. 16, julio-diciembre, 2014, pp. 13-29. México: Instituto Tecnológico y de Estudios Superiores de Monterrey Campus Ciudad de México. Recuperado de: http://www.redalyc.org/pdf/1411/141132947001.pdf +

Gardner, H. (2005). Múltiples lentes sobre la mente. Separata. Sinéctica. Trabajo presentado en la Conferencia de Expo Gestión, Bogotá, Colombia.

Giri Goswami, T., Pareek, R., Giri, P., \& Giri, S. (2018). Workplace happiness and productivity: A conceptual analysis. MAER, Management \& Applied Economics Review, 24, 82-89. Retrieved from http://search.ebscohost.com/login.aspx?di rect=true\&db=bsx\&AN=133361429\&lang=es\&site=eds-live\&scope=site

Guriev, S., \& Melnikov, N. (2018). Happiness convergence in transition countries. Journal of Comparative Economics, 46(3), 683-707. https://doi. org/10.1016/j.jce.2018.07.003

Hernández, G. (2016). Procrastinación académica, motivos de procrastinación y bienestar psicológico en alumnos de ingeniería industrial de una universidad de Trujillo. Tesis de Grado. Universidad Privada del Norte, Perú.

Kok Fong See, \& Siew Hwa Yen. (2018). Does happiness matter to health system efficiency? A performance analysis. Health Economics Review, Vol 8, Iss 1, Pp 1-10 (2018), (1), 1. https://doi.org/10.1186/s13561-018-0214-6

Laczniak, G. R., \& Santos, N. J. C. (2018). Gross National Happiness (GNH). Journal of Macromarketing, 38(3), 331-340. https://doi.org/10.1177/0276146718787600 
Marques, C., Maria, d. C., Ceinos, C., Silva, A. D., \& Pérez, M. (2018). Satisfacción con la vida en estudiantes universitarios. Psicología Desde El Caribe, 35(3) Retrieved from https://search.proquest.com/docview/2116449673?accountid=45097

Port, A. P., Dianniffer Aparecida, d. O., de, O. B., \& Thaline da, C. M. (2018). Variables associated with the meaning of life. Revista Da Abordagem Gestáltica, 24(1), 35-43. Retrieved from https://search.proquest.com/ docview/2024255561?accountid=45097

Pulido-Acosta, F., \& Herrera-Clavero, F. (2018). Predictors of happiness and academic performance in a multicultural sample of adolescents. Revista de Estudios e Investigación En Psicología y Educación, Vol 5, Iss 1, Pp 9-18 (2018), (1), 9. https://doi.org/10.17979/reipe.2018.5.1.2991

Ramírez, P.y Fuentes, C. (2013). Felicidad y rendimiento académico:Efectomoderador de la felicidad sobre indicadores de selección y rendimiento académico de alumnos de ingeniería comercial. Universidad Católica del Norte. Coquimbo, Chile.

Rojas, E. (2017). La Felicidad y su relación con el Compromiso Organizacional de los trabajadores de la I.E Everardo Zapata Santillana - Trujillo 2017. Tesis para Licenciatura en Administración. Universidad Cesar Vallejo, Perú.

Rokeach, M. (1979). Understanding Human Values. Individual al Societal. New York, USA: The Free Press.

Rosales, A. (2017). Autoeficacia y felicidad en estudiantes universitarios de la carrera de psicología de una universidad privada de Lima Sur. Tesis para Licenciado en Psicología. Universidad Autónoma del Perú.

Sánchez-López,D.,León-Hernández,S.yBarragán-Velásquez,C.(2015).Correlación de Inteligencia Emocional con Bienestar Psicológico y Rendimiento Académico en Alumnos de Licenciatura. Investigación en Educación Médica. 4(15), 126-132 http://dx.doi.org/10.1016/j.riem.2015.04.002

Sartre, J. (2006). El existencialismo es un humanismo. Universidad Nacional Autónoma de México. Consultado en https://books.google.com.pe/books? $\mathrm{hl}=\mathrm{es} \& \mathrm{Ir}=\& \mathrm{id}=3 \mathrm{yy} 8 \mathrm{~F} 1 \mathrm{pPfr} 8 \mathrm{C} \& \mathrm{oi}=\mathrm{fnd} \& \mathrm{pg}=\mathrm{PA}$ \% \&dq=existencialismo\&ots=Z6 wcpdVRa0\&sig=-jul4vmt_90A18io4Dj3ODCrvr0\#v=onepage\&q=existenciali smo\&f $=$ false

Seligman, M. (2017). La auténtica felicidad. España: Ediciones B.

Ugarriza, N. (2005). Depresión, expectativas frente al futuro y rendimiento académico en estudiantes universitarios del ciclo inicial. Informe final. (Inédito) Lima: Centro de Investigación de la Universidad Ricardo Palma CIURP.

UIC. Foro multidisciplinario de la universidad intercontinental. (2013) Psicología de la felicidad. Consultado en http://www.biblioteca.uic.edu.mx/Revistas/ forouic/UIC27.pdf 